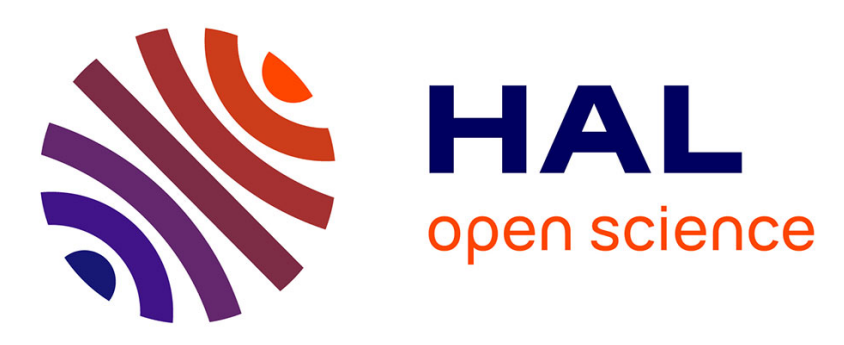

\title{
Model for random packing of polydisperse frictionless spheres
}

Eric I. Corwin, Maxime Clusel, Alexander Siemens, Jasna Brujic

\section{To cite this version:}

Eric I. Corwin, Maxime Clusel, Alexander Siemens, Jasna Brujic. Model for random packing of polydisperse frictionless spheres. Soft Matter, 2010, 6 (13), pp.2949-2959. 10.1039/C000984A . hal00641690

\section{HAL Id: hal-00641690 \\ https://hal.science/hal-00641690}

Submitted on 16 Nov 2011

HAL is a multi-disciplinary open access archive for the deposit and dissemination of scientific research documents, whether they are published or not. The documents may come from teaching and research institutions in France or abroad, or from public or private research centers.
L'archive ouverte pluridisciplinaire HAL, est destinée au dépôt et à la diffusion de documents scientifiques de niveau recherche, publiés ou non, émanant des établissements d'enseignement et de recherche français ou étrangers, des laboratoires publics ou privés. 


\title{
Model for random packing of polydisperse frictionless spheres $\dagger$
}

\author{
Eric I. Corwin, $₫$ Maxime Clusel, $₫ \oint$ Alexander O. N. Siemens $\llbracket$ and Jasna Brujić*
}

\author{
Received 15th January 2010, Accepted 29th March 2010 \\ First published as an Advance Article on the web 3rd June 2010 \\ DOI: 10.1039/c000984a
}

\begin{abstract}
We propose a statistical model for the random packing of frictionless polydisperse spheres in which the complexity of the global packing is distilled into a local stochastic process. We simplify the problem by considering the "granocentric" point of view of a single particle in the bulk, thereby reducing random packing to the assembly of nearest neighbours, followed by a random choice of contacts among them. The model is based on only two parameters, the available solid angle around each particle and the ratio of contacts to neighbors, which are both directly obtainable from experiments or simulations. As a result, the model analytically predicts the microscopic distributions of nearest neighbours and contacts, the local density fluctuations as well as the global density of the packing. We find that this granocentric view captures the essential properties of the polydisperse emulsion packing. This model suggests a general principle of organization for random packing and provides a statistical tool for quantifying the effect of the particle size distribution on the geometry of random packing in a variety of contexts of industrial relevance.
\end{abstract}

\section{Introduction}

Particulate packings occur ubiquitously in nature, from grains in silos to oil droplets in mayonnaise. How a set of particles randomly assembles into a mechanically stable packing is a problem that has puzzled scientists for centuries. ${ }^{1}$ Above a given density, the system undergoes a jamming transition ${ }^{2,3}$ to a mechanically stable structure. Several theoretical approaches have been proposed to understand the physics of this transition. For example, random packing of monodisperse spheres has been considered in analogy with the glass transition ${ }^{4,5}$ - experimentally, ${ }^{6-8}$ computationally, ${ }^{9,10}$ and theoretically. ${ }^{11-13}$ Moreover, a statistical mechanics framework for jammed matter has been used to relate the probability distributions of microscopic quantities in the packing to macroscopic properties of the system. ${ }^{14-17}$ Despite these important advances, a governing principle for the random organization of particles that predicts their packing geometry remains unknown. The problem appears even more challenging as soon as one advances to real particulate systems found in nature, which are polydisperse, ${ }^{18}$ frictional, ${ }^{19}$ deformable ${ }^{20}$ and comprised of different shapes. ${ }^{21}$

In this paper, we develop a statistical framework termed the "granocentric" model to account for the effects of polydispersity on packing properties. The granocentric model attempts to give a physical intuition for the microscopic mechanism behind the

New York University, Department of Physics and Center for Soft Matter Research, 4 Washington Place, New York, NY, 10003, USA. E-mail: ec81@nyu.edu; jb2929@nyu.edu

$\dagger$ This paper is part of a Soft Matter themed issue on Granular and jammed materials. Guest editors: Andrea Liu and Sidney Nagel.

\$ Both authors contributed equally to this work

$\S$ Present address: Institut Laue-Langevin, Theory group, 6 rue Jules Horowitz, 38042, Grenoble Cedex, France. E-mail: maxime.clusel@ill.eu ๆ Present address: Granular \& Disordered Media, Kamerlingh Onnes Laboratory, Leiden University, P.O. Box 9504. 2300 RA, Leiden, The Netherlands. E-mail: siemens@physics.leidenuniv.nl random variety of local configurations present in the packing and to relate the statistics of these local configurations to global properties of the packing, such as its density. This model was previously shown to describe the geometric properties of random packings of polydisperse emulsion droplets. ${ }^{22}$ This study was limited to the size distribution of the emulsion fabrication, i.e. a log-normal distribution with a typical mean radius of $\sim 3 \mu \mathrm{m}$ and a standard deviation of $\sim 0.7 \mu \mathrm{m}$. The goal of the present article is to go beyond this specific experimental verification in order to study the limits of validity of the model using numerical simulations to create random packings of spheres of controlled size distributions.

\section{A. Background}

Since real particulate systems are polydisperse, accounting for the effects of their size distribution on packing is of industrial relevance for grain storage, drying of paints, and construction of porous membranes to name but a few examples. While in the case of monodisperse spheres all the randomness comes from the positional disorder of the constituents, the size distribution in polydisperse systems introduces an additional source of randomness into the packing structure. To capture the effect of polydispersity, ${ }^{18,23,24}$ several models have been proposed to estimate the global packing fraction based on the particle size distribution, either using simplified packing models, ${ }^{25,26}$ linear packing models ${ }^{27,28}$ or a mixture packing model. ${ }^{28}$

Especially noteworthy is a simple geometric model proposed in the early 1970s by Dodds to predict the packing fraction of polydisperse packings by enumerating all the possible local arrangements of spheres of different sizes. This model was first developed for $2 \mathrm{~d}$ packings of disks ${ }^{29,30}$ and later extended to $3 \mathrm{~d}$ packings of polydisperse spheres. ${ }^{31}$ Using this local point of view, Dodds developed a combinatorial approach to estimate the packing fraction as well as the distribution of the number of contacts. This model is based on the assumption that each sphere 
touches its neighbours - an assumption that is not valid for real packings and requires a rescaling of the model predictions to match experimental data. ${ }^{32}$ Nevertheless, Dodds' approach presented a significant leap in understanding packings by demonstrating that a simple local model can capture certain properties of the packing organisation. The granocentric model developed in this paper builds on Dodds' idea of a local statistical description of the packing and provides a more complete picture of the packing structure by providing access to the distributions of the numbers of contacts and neighbours, as well as the local and global packing fractions. This is made possible because our model does not make the assumption that all particles are in contact with their neighbours.

\section{B. Organization of the paper}

The remainder of this article is organised as follows. Section II discusses the organization of polydisperse packings, presents the physically relevant quantities under consideration and describes how they can be measured both in experiments and numerical simulations. Section III lists the numerical methods employed to simulate frictionless polydisperse packings. Section IV presents the model in detail and comments on its results. Section V concerns an extension of the model to local and global packing fraction. Section VI tests the model and probes its applicability, while Section VII offers a discussion of the model's success in describing experiments.

\section{Polydisperse packings}

A jammed packing of polydisperse spheres is achieved by sufficiently increasing the global density to bring the particles in contact with one another such that the isostatic condition is satisfied. ${ }^{33,34}$ In the case of frictionless spheres, as considered in this article, isostaticity is reached when the average number of contacts per particle is larger than or equal to $2 d$, where $d$ is the spatial dimension.||The jamming transition is defined as the point where the packing is marginally stable, so that for three-dimensional frictionless packings the mean number of contacts per particle is 6 .

Once a jammed packing has been created, its geometry is described completely by specifying the centerpoint positions and the sizes of every particle. This full microscopic description of the packing geometry can then be used to characterize the local environment around each particle by collecting statistics over all the particles in the system. This allows for the measurement of quantities such as the probability distributions of the number of contacts, number of neighbours and local volume fraction around particles of a given size in the packing. In turn these distributions can be used to calculate global quantities, such as the packing density. In this section we define precisely the distributions of the number of contacts, number of neighbours and volume fraction, and indicate how they can be measured in experiments and numerical simulations. This begins with a tessellation of space to analyze the packing structure, explained next.

\| Note that the presence of friction changes the local force balance and therefore modifies the isostatic condition, which becomes $\langle z\rangle=d+1$.

\section{A. Packing geometry via the Johnson-Mehl tessellation}

A systematic way to analyze a packing is to define a local cell around each particle by tessellating the system. In the present work we use the Johnson-Mehl tessellation, also referred to as the navigation map. ${ }^{35-37}$ The cell around a given particle in this tessellation is defined as the region which is closer to the surface of this particle than any other particle's surface. For monodisperse packings this reduces to the usual Voronoi construction, ${ }^{38}$ defined as the region closer to a given particle's centerpoint than any other particle's centerpoint. For polydisperse packings the cell boundaries are no longer planes but rather are portions of hyperboloids, ${ }^{37}$ shown in Fig. 1.

\section{B. Contacts, neighbours and local packing fraction}

The Johnson-Mehl tessellation permits us to define three quantities that describe the local environment of each particle - the number $n$ of neighbours surrounding a given particle, the subset of $z$ contacts that contribute to its mechanical rigidity and the local packing fraction $\phi_{\text {local }}=V_{\text {particle }} / V_{\text {cell }}$, where $V_{\text {particle }}$ is the volume of the particle and $V_{\text {cell }}$ the volume of its Johnson-Mehl cell. This last quantity is the packing efficiency at the single particle level, and can be measured directly. Two particles are said to be neighbours if they have a common interface in the Johnson-Mehl tessellation, while they are said to be in contact if their surface-to-surface distance is exactly zero. For instance, the particles coloured in green and blue in Fig. 1 are neighbours of the central black particle, but only the blue particle is also in contact. The neighbours participate in creating the local geometric structure of a jammed system without contributing directly to the local mechanical stability of a given central particle.

Experimentally, imaging a refractive index-matched, jammed emulsion identifies the contacts around a particle using Nile red dye, which has an enhanced fluorescence at the contact points. ${ }^{39}$ The determination of the set of neighbours is done by tessellating the reconstructed images via the navigation map. Whereas the

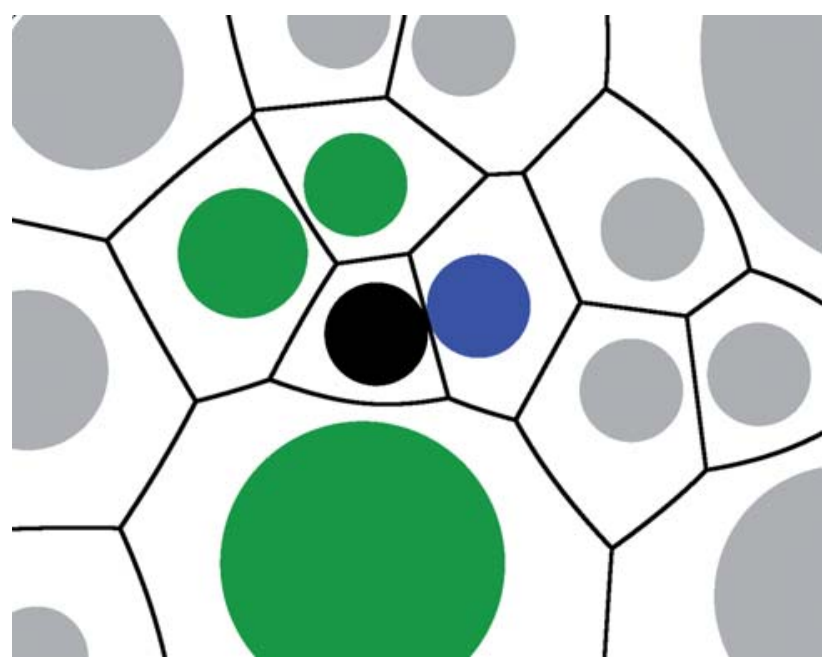

Fig. 1 Example of Johnson-Mehl tessellation for a set of polydisperse disks in $2 \mathrm{~d}$. Note that the black particle has four neighbours (green and blue) of which one is in contact (blue). 
definition of contacts is sensitive to the spatial resolution of the microscope, neighbours are unambiguously defined in the packing.

Once we have determined the Johnson-Mehl tessellation we can compute the mean number of contacts per particle, $\langle z\rangle$, the probability density of contacts $P_{\mathrm{c}}(z)$, as well as the conditional contact number probability density around all particles of radius $r_{\mathrm{c}}, P_{\mathrm{c}}\left(z \mid r_{\mathrm{c}}\right)$, with the following relations:

$$
\begin{gathered}
P_{\mathrm{c}}(z)=\int \mathrm{d} r P_{\mathrm{c}}(z \mid r) P(r), \\
\langle z \mid r\rangle=\sum_{z=0}^{\infty} z P_{\mathrm{c}}(z \mid r), \\
\langle z\rangle=\sum_{z=0}^{\infty} z P_{\mathrm{c}}(z) .
\end{gathered}
$$

The isostatic limit for mechanical stability requires that $\langle z\rangle \geq 6$. $^{33}$ This imposes a global constraint on the probability density of $P_{\mathrm{c}}(z)$. However, the specific shapes of this probability density depend on the size distribution of the particles.

In like fashion, we can compute the mean number of neighbours per particle, $\langle n\rangle$, the probability density of neighbours $P_{\mathrm{n}}(n)$, as well as the conditional neighbour number probability density around all particles of radius $r_{\mathrm{c}}, P_{\mathrm{n}}\left(n \mid r_{\mathrm{c}}\right)$, with the following relations:

$$
\begin{array}{r}
P_{\mathrm{n}}(n)=\int \mathrm{d} r P_{\mathrm{n}}(n \mid r) P(r), \\
\langle n \mid r\rangle=\sum_{n=0}^{\infty} n P_{\mathrm{n}}(n \mid r), \\
\langle n\rangle=\sum_{n=0}^{\infty} n P_{\mathrm{n}}(n) .
\end{array}
$$

Note that there is no constraint on the mean number of neighbours per particle.

Apart from the probability densities of neighbours and contacts, the Johnson-Mehl tesselation also allows us to measure the probability density of local packing fractions, defined as the volume of the particle divided by the volume of its cell. The global packing fraction, defined as $\phi_{\text {global }}=V_{\text {matter }} / V_{\text {sample }}$, can be computed without using a particular tessellation since

$$
V_{\text {matter }}=\sum_{k=1}^{N} V_{\text {particle }}\left(r_{k}\right),
$$

where $N$ is the total number of particles in the sample and $V_{\text {particle }}\left(r_{k}\right)$ is the volume of the $k^{\text {th }}$ particle. However, it should be stressed that the global packing fraction $\phi_{\text {global }}$ is not $\left\langle\phi_{\text {local }}\right\rangle=\int_{0}^{\infty} \mathrm{d} \phi_{\text {local }} \phi_{\text {local }} P_{\mathrm{v}}\left(\phi_{\text {local }}\right)$.

\section{Numerical methods}

In order to test the predictions of the granocentric model, we need to compare it to a well controlled system in which we can easily and arbitrarily vary the particle size distribution. Doing this experimentally poses numerous challenges; however, numerical simulations are well suited to the creation of frictionless polydisperse packings of spherical particles. Simulations are done using the Large-scale Atomic/Molecular Massively Parallel Simulator (LAMMPS) ${ }^{40}$ modified to allow for arbitrarily defined polydispersities. Spherical frictionless particles, which interact through Hertzian contact forces, are simulated. We followed a simplified version of the infinite temperature quench technique presented in ref. 41 to create jammed packings. Each simulation starts with a fixed number of particles $(N=10000)$ whose positions are distributed randomly within a cubic box of side length $L$ and periodic boundary conditions. Doing so approximates an infinite temperature condition. The particles' radii are drawn from a given $P(r)$ such that the global packing fraction $\phi$ is equal to a preselected value. The temperature is then quenched close to zero and nearly all of the energy in the system is removed by allowing particles to move away from one another along the path of steepest descent. Since we only consider jammed packings near the jamming threshold it was sufficient for our purposes to repeat this process over and over, increasing the packing fraction from below the jamming threshold in small increments $(\Delta \phi=0.001)$ until a mechanically stable packing was found. Additional simulation results for a range of distributions performed with this technique may be found in the supplementary materials to our previous paper. ${ }^{22}$

\section{The granocentric model}

\section{A. Granocentric point of view}

We propose a local model for random packing of polydisperse spheres. The model describes the packing as seen from the "granocentric" point of view of a particle in the bulk of the packing. From this perspective, the formation of a packing can be seen in the following way. Starting with an initially dilute, unjammed system and slowly increasing the particle density, a given central particle will see more and more particles filling its neighbourhood as defined by the Johnson-Mehl tessellation. Eventually, all the space around this particle is occupied by neighbours, such that it is impossible to fit an additional particle. As the density is further increased some of the neighbours must come into contact with the central particle, such that the particle is mechanically constrained and the jamming threshold is reached. These two local processes provide the physical intuition for the development of a granocentric model for packing. Below we first explain the underlying assumptions behind each of these two steps, then define the mathematical framework in the next subsections.

Physically, the limitation of space around a given particle is due to the interparticle interaction preventing neighbouring particles from overlapping. Clearly the space available around each particle is determined by the arrangement of its neighbours. In our model we introduce the solid angle $\Omega_{\max }\left(r_{\mathrm{c}}\right)$ available around a sphere of radius $r_{\mathrm{c}}$, as an effective parameter to describe this geometrical congestion. These sterical effects mean that $\Omega_{\max }\left(r_{\mathrm{c}}\right)$ is not simply $4 \pi$ as one would expect for the total solid angle around a sphere. Its precise value will depend on the polydispersity and the local packing structure. We will demonstrate that the value of $\Omega_{\max }\left(r_{\mathrm{c}}\right)$ can be self-consistently determined for a given packing. 
We model the formation of the shell of neighbours by the random addition of particles of radii $r$, characterised by the solid angle $\Omega\left(r_{\mathrm{c}}, r\right)$ it subtends on the central particle of radius $r_{\mathrm{c}}$. We make the assumption that the system is statistically homogeneous and isotropic, such that each particle in the packing sees locally the same type of polydispersity as any other particle in the packing. (The limits of this assumption in real polydisperse packing will be discussed in section VI.) We therefore model the formation of the neighbour shell by the first passage problem of a one-dimensional random walk. The number of neighbours is then defined as the number of steps the walk makes until the total solid angle covered by the neighbours reaches, without crossing, $\Omega_{\max }\left(r_{\mathrm{c}}\right)$. We then make the additional assumption that there is no correlation between the successive steps of this random walk: a particle is added to the shell of neighbours if there is enough space left, independently of the particles previously added.

In the second step, we model the selection of each contact among the set of neighbours as an independent process. The validity of this assumption will be justified a posteriori by the success of the model in describing measurements. The independent choice among neighbours means that we do not include collective behaviour: the fact that one neighbour is in contact does not bias the choice as to whether another will be. Based on this assumption the determination as to whether a neighbour is in contact is a Bernoulli trial. Thus, the probability of having $z$ contacts among $n$ neighbours about a central particle of radius $r_{\mathrm{c}}$ is given by the binomial distribution with success probability $p\left(r_{\mathrm{c}}\right)$.

\section{B. Distribution of solid angle}

In the granocentric model the formation of the set of neighbours is represented by the summation of the solid angles they subtend on a central particle. The relevant distribution characterising the polydispersity is then the two-point distribution of solid angle $\Omega$ around a central particle of radius $r_{\mathrm{c}}$. For a given central particle radius $r_{\mathrm{c}}$, the corresponding solid angle distribution can be obtained from the radius distribution by a change of variable as follows. Let $\mathrm{S}_{\mathrm{c}}$ be a sphere of radius $r_{\mathrm{c}}$. A sphere of radius $r$ in contact with $\mathrm{S}_{\mathrm{c}}$ will subtend a solid angle

$$
\Omega\left(r_{\mathrm{c}}, r\right)=2 \pi\left(1-\frac{1}{1+\frac{r}{r_{\mathrm{c}}}} \sqrt{1+2 \frac{r}{r_{\mathrm{c}}}}\right) \in 0,2 \pi .
$$

Note that $\Omega\left(r_{\mathrm{c}}, r\right)$ is bounded between 0 and $2 \pi$ by construction. The distribution of $\Omega\left(r_{\mathrm{c}}, r\right)$ can be obtained from $P(r)$ following the steps presented in Appendix A. Let $f_{\mathrm{c}}(\Omega)$ be the probability density of a solid angle $\Omega\left(r_{\mathrm{c}}, r\right)$ given the sphere $\mathrm{S}_{\mathrm{c}}$. We then have

$$
f_{\mathrm{c}}(\Omega)=P(r)\left(\frac{\mathrm{d} \Omega}{\mathrm{d} r}\left(r_{\mathrm{c}}, r\right)\right)^{-1},
$$

leading to

$$
\begin{aligned}
f_{\mathrm{c}}(\Omega)= & r_{\mathrm{c}} \frac{1+\Omega / 2 \pi(2-\Omega / 2 \pi)+2 \sqrt{\Omega / 2 \pi(2-\Omega / 2 \pi)}}{(1-\Omega / 2 \pi)^{3} \sqrt{\Omega / 2 \pi(2-\Omega / 2 \pi)}} \\
& \times P\left[r_{\mathrm{c}} \frac{\Omega / \pi-(\Omega / 2 \pi)^{2}+\sqrt{\Omega / \pi-(\Omega / 2 \pi)^{2}}}{(1-(\Omega / 2 \pi))^{2}}\right] .
\end{aligned}
$$

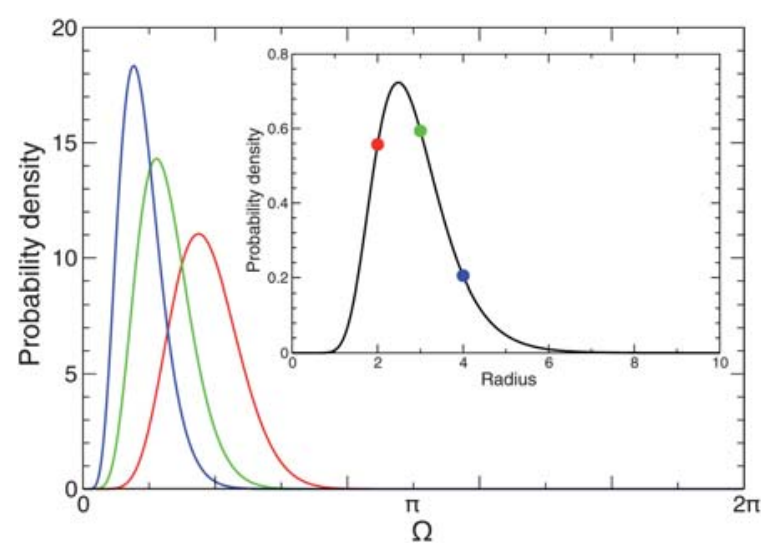

Fig. 2 Examples of solid angle distributions obtained from a given $P(r)$ for three different central particle radii, indicated by the colour of the markers in the inset.

This relation, illustrated in Fig. 2, is nonlinear and maps a distribution defined on $\mathbb{R}^{+}$to a new distribution defined on $0,2 \pi$.

\section{Distribution of number of neighbours}

Let $\mathrm{S}_{\mathrm{c}}$ be a sphere of radius $r_{\mathrm{c}}$ in the packing. We pick a sequence of spheres characterised by the list of their radii $\left\{r_{k}\right\}$, or equivalently by the list of the solid angles they subtend on $\mathrm{S}_{\mathrm{c}},\left\{\Omega_{k}\right\}$. The number of nearest neighbours to this sphere $S_{c}$ is then defined as the random number of spheres $n$ drawn from this sequence that can be placed about $\mathrm{S}_{c}$, such that the total solid angle occupied by the neighbours is as close as possible to $\Omega_{\max }\left(r_{\mathrm{c}}\right)$, without exceeding it. Formally, the number of neighbours is defined by

$$
\sum_{k=1}^{n} \Omega_{k} \leq \Omega_{\max } \text { and } \sum_{k=1}^{n+1} \Omega_{k}>\Omega_{\max },
$$

or equivalently,

$$
\exists \Omega \in\left[0, \Omega_{\max }\right], \Omega=\sum_{k=1}^{n} \Omega_{k} \text { and } \Omega_{n+1}>\Omega_{\max }-\Omega .
$$

From the last definition it is easy to see that $P_{\mathrm{n}}\left(n \mid r_{\mathrm{c}}\right)$, the conditional probability density to have $n$ neighbours around a sphere of radius $r_{\mathrm{c}}$, is given by the convolution product

$$
P_{\mathrm{n}}\left(n \mid r_{\mathrm{c}}\right)=\int_{0}^{\Omega_{\max }} \mathrm{d} \Omega R_{n}(\Omega) F_{r_{\mathrm{c}}}\left(\Omega_{\max }-\Omega\right),
$$

$$
P_{\mathrm{n}}\left(n \mid r_{\mathrm{c}}\right)=\left(R_{n} \star F_{r_{\mathrm{c}}}\right)\left(\Omega_{\mathrm{max}}\right),
$$

where $R_{n}(\Omega)$ is the probability to reach $\Omega$ after exactly $n$ steps, $F_{r_{\mathrm{c}}}(w)=\int_{w}^{\infty} \mathrm{d} u f_{\mathrm{c}}(u)$, and $\star$ denotes the convolution product.

Taking the Laplace transform with respect to $\Omega_{\max }$, we obtain:

$$
\mathscr{L}\left[P_{\mathrm{n}}\left(n \mid r_{\mathrm{c}}\right)\right](s)=\mathscr{L}\left[R_{n}\right](s) \mathscr{L}\left[F_{r_{\mathrm{c}}}\right](s),
$$

where $s$ is the conjugate variable of $\Omega_{\max }$ through the Laplace transform and $\mathscr{L}$ denotes the Laplace transform operator. Using 


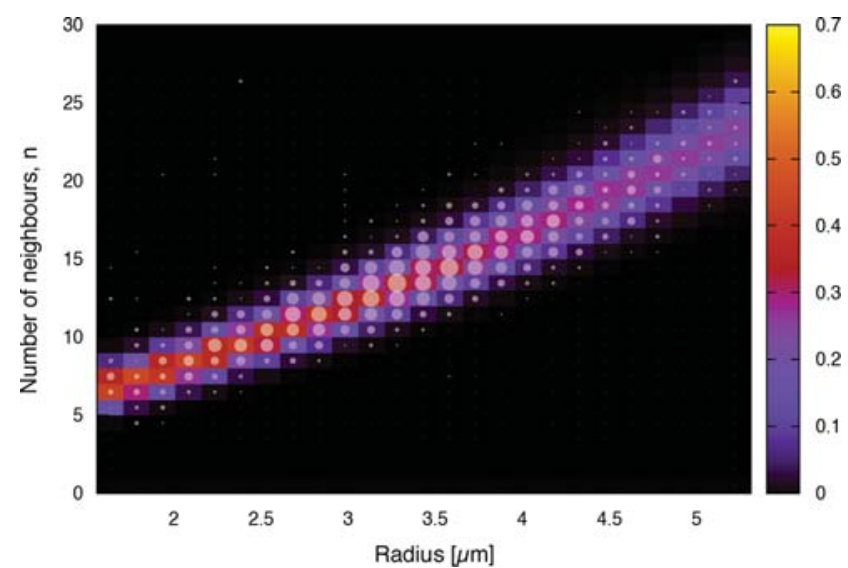

Fig. 3 Representative conditional number of neighbours distribution. The value of $P\left(n \mid r_{\mathrm{c}}\right)$ is given by the intensity scale shown on the right. The size of the dots corresponds to the distribution of the number of neighbours in an oil-in-water emulsion, in good agreement with the model predection. $^{22}$

basic properties of the Laplace transform, and introducing the notation

$$
\hat{f}_{\mathrm{c}}(s)=\mathscr{L}\left[f_{\mathrm{c}}\right](s),
$$

we then have

$$
\mathscr{L}\left[F_{r_{\mathrm{c}}}\right](s)=\frac{1-\hat{f}_{\mathrm{c}}(s)}{s} .
$$

Moreover, under the assumption that the solid angles around the central particle are independent and identically distributed random variables, we have

$$
\mathscr{L}\left[R_{n}\right](s)=\left[\hat{f}_{\mathrm{c}}(s)\right]^{n},
$$

so that finally

$$
\mathscr{L}\left[P_{\mathrm{n}}\left(n \mid r_{\mathrm{c}}\right)\right](s)=\frac{1-\hat{f}_{\mathrm{c}}(s)}{s}\left[\hat{f}_{\mathrm{c}}(s)\right]^{n},
$$

$$
P_{\mathrm{n}}\left(n \mid r_{\mathrm{c}}\right)=\mathscr{L}^{-1}\left[\frac{1-\hat{f}_{\mathrm{c}}(s)}{s}\left[\hat{f}_{\mathrm{c}}(s)\right]^{n}\right]\left(\Omega_{\max }\right),
$$

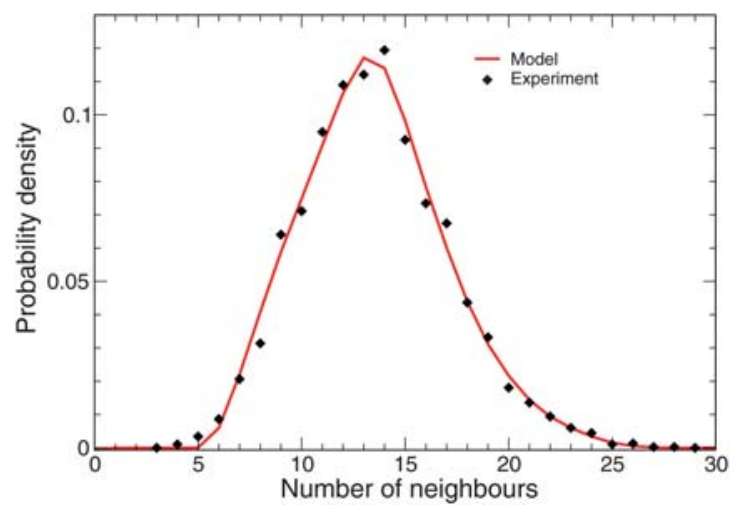

Fig. 4 Representative probability distribution of number of neighbours predicted by model compared to observed distribution. where $\mathscr{L}^{-1}$ denotes the inverse Laplace transform operator. This prediction of $P\left(n \mid r_{\mathrm{c}}\right)$ is presented along with experimental data in Fig. 3. Note that the dependence on the central particle radius comes from the fact that the solid angle distribution depends on $r_{\mathrm{c}}$, as seen in eqn (10). In general it is not possible to compute analytically the inverse Laplace transform in eqn (20), but this inversion can be done numerically, for example using Mathematica and a package for the numerical inversion of the Laplace transform. ${ }^{42}$ The distribution given in eqn (19) can be integrated over the particle radius distribution to obtain the overall number of neighbour distributions

$$
P_{\mathrm{n}}(n)=\int \mathrm{d} r_{\mathrm{c}} P\left(r_{\mathrm{c}}\right) P_{\mathrm{n}}\left(n \mid r_{\mathrm{c}}\right),
$$

as presented in Fig. 4.

\section{Estimation of $\boldsymbol{\Omega}_{\max }\left(r_{c}\right)$}

Eqn (20) gives a way to estimate the parameter $\Omega_{\max }\left(r_{\mathrm{c}}\right)$ from measurements of the mean number of neighbours as a function of $r_{\mathrm{c}}$. Indeed, we have

$$
\left\langle n \mid r_{\mathrm{c}}\right\rangle=n_{c}\left(\Omega_{\max }\right)=\sum_{n=0}^{\infty} n P_{\mathrm{n}}(n),
$$

$$
\hat{n}_{c}(s)=\sum_{n=0}^{\infty} n \frac{1-\hat{f}_{c}(s)}{s}\left[\hat{f}_{c}(s)\right]^{n}=\frac{1}{s} \frac{\hat{f}_{c}(s)}{1-\hat{f}_{c}(s)},
$$

$$
n_{c}\left(\Omega_{\max }\right)=\mathscr{L}^{-1}\left[\frac{1}{s} \frac{\hat{f}_{c}(s)}{1-\hat{f}_{c}(s)}\right]\left(\Omega_{\max }\right) .
$$

By numerically solving eqn (24) the parameter $\Omega_{\max }\left(r_{\mathrm{c}}\right)$ can be self-consistently determined by experimental measurements of the mean number of neighbours. In principle, the available solid angle could depend on the central particle radius $r_{\mathrm{c}}$. However, we can also define the averaged available solid angle

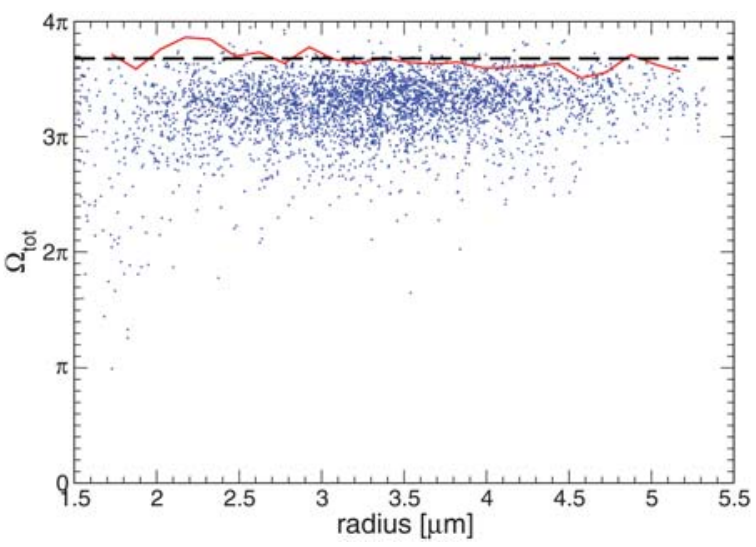

Fig. 5 Example of values obtained for $\Omega_{\max }$ using eqn (24) and experimental measurements of number of neighbours as a function of $r_{\mathrm{c}}$. The red curve corresponds to the values obtained by solving eqn (24), while the dashed line corresponds to $\left\langle\Omega_{\max }\right\rangle \simeq 3.68 \pi$. Note that in this particular case the dependence of $\Omega_{\max }\left(r_{\mathrm{c}}\right)$ on $r_{\mathrm{c}}$ is negligible. The dots correspond to the total occupied solid angle as measured in an oil-inwater emulsion. ${ }^{22}$ 
$\left\langle\Omega_{\max }\right\rangle=\int \mathrm{d} r_{\mathrm{c}} P\left(r_{\mathrm{c}}\right) \Omega_{\max }\left(r_{\mathrm{c}}\right)$. Fig. 5 presents an example of the values of $\Omega_{\max }\left(r_{\mathrm{c}}\right)$ and $\left\langle\Omega_{\max }\right\rangle$ obtained from an experimental packing of oil-in-water droplets. In this particular case the dependence of $\Omega_{\max }$ on $r_{\mathrm{c}}$ is negligible, $\Omega_{\max }\left(r_{\mathrm{c}}\right) \simeq\left\langle\Omega_{\max }\right\rangle \simeq$ $3.68 \pi$. The fact that the value of $\Omega_{\max }$ is smaller than the available solid angle around a sphere in empty space $(4 \pi)$ characterizes the local cluttering around the central particle.

\section{E. Distribution of number of contacting neighbours}

Once we have characterized the geometry of the packing around a central particle as described by the distribution of the number of neighbours, we next turn to the distribution of the number of contacting neighbours. This subset of the neighbours is responsible for the mechanical stability of the packing. We make the assumption that the selection of a set of contacting neighbours is a local process neglecting all possible correlation: a neighbour is or is not also a contact independently of whether any other neighbours are contacts. Therefore the selection of contacts among neighbours must reduce to a succession of Bernoulli trials, each with a probability of success $p\left(r_{\mathrm{c}}\right)$. Note that this probability for a neighbour to be in contact can in principle depend on the central particle radius.

Thus, the choice of a set of contacting neighbours can be seen as a second random process choosing $z$ contacts among the $n$ neighbours with a probability $p\left(r_{\mathrm{c}}\right)$. Acting under this assumption we can obtain $P\left(z \mid r_{\mathrm{c}}\right)$, the distribution of the number of contacts around a particle of radius $r_{\mathrm{c}}$. Therefore we have:

$$
P_{\mathrm{c}}\left(z \mid r_{\mathrm{c}}\right)=\sum_{n=z}^{\infty} C_{n}^{z} p\left(r_{\mathrm{c}}\right)^{z}\left(1-p\left(r_{\mathrm{c}}\right)\right)^{n-z} P_{\mathrm{n}}\left(n \mid r_{\mathrm{c}}\right),
$$

where $C_{n}{ }^{2}$ is the binomial coefficient. If we take the Laplace transform of $P_{\mathrm{c}}\left(z \mid r_{\mathrm{c}}\right)$ with respect to $\Omega_{\max }$ we find:

$$
\mathscr{L}\left[P_{\mathrm{c}}\left(z \mid r_{\mathrm{c}}\right)\right](s)=\sum_{n=z}^{\infty} C_{n}^{z} p^{z}(1-p)^{n-z} \frac{\left(1-\hat{f}_{\mathrm{c}}(s)\right) \hat{f}_{\mathrm{c}}(s)^{n}}{s},
$$

where $s$ is again the conjugate variable of $\Omega_{\max }$ through the Laplace transform. Since $f_{\mathrm{c}}$ is a probability density, we know that $(1-p) \hat{f}_{\mathrm{c}}(s)<1$, and the series can be summed to obtain the final expression for the Laplace transform of $P_{c}\left(z \mid r_{\mathrm{c}}\right)$ :

$$
\mathscr{L}\left[P_{\mathrm{c}}\left(z \mid r_{\mathrm{c}}\right)\right](s)=\frac{p^{z}}{\left[1-(1-p) \hat{f_{\mathrm{c}}}(s)\right]^{z+1}} \mathscr{L}\left[P_{\mathrm{n}}\left(z \mid r_{\mathrm{c}}\right)\right](s) .
$$

This expression can be inverted numerically to obtain $P_{\mathrm{c}}\left(z \mid r_{\mathrm{c}}\right)$.

\section{F. Estimation of $p\left(r_{c}\right)$}

The value of the contact probability $p\left(r_{\mathrm{c}}\right)$ can be determined using experimental or numerical data. Eqn (25) implies the simple relation:

$$
p\left(r_{\mathrm{c}}\right)=\frac{\left\langle z \mid r_{\mathrm{c}}\right\rangle}{\left\langle n \mid r_{\mathrm{c}}\right\rangle}
$$

Note that the model makes no a priori assumptions on the nature of the dependence of contact probability on the central particle radius $r_{\mathrm{c}}$.

\section{Global and local packing fraction}

In the previous section we have focused on the statistical description of the packing's connectivity. In this section we will see that this model can be complemented by a definition of a local cell to gain access to the statistics of local packing fraction. This quantity gives information on the packing efficiency at the single particle level. ${ }^{16,43,44}$ Knowing the local cells additionally gives us access to the global packing fraction.

\section{A. Definition of a local cell}

The Johnson-Mehl tessellation gives a precise definition of a local cell for each particle in a packing. However the properties of a cell, in particular its volume, depend on the position of the neighbours around the central particle: since the model thus far does not incorporate this type of information, we need to extend it to compute the volume of a cell. To this end, we define an effective cell around each particle in the packing, using only information on the number of neighbours and the number of contacts. Note that the definition of the local cell is somewhat arbitrary and one could imagine a number of alternate definitions. What is important is to capture the essential physical features one expects from a local cell: 1) for a given central particle, the cell volume must increase with the number of neighbours, and 2) for a given number of neighbours, the cell volume increases with the fraction of non-contacting neighbours.

Feature one will be satisfied by defining the volume of a cell as the sum of volume contributions $V^{*}\left(r_{\mathrm{c}}, r_{k}, \delta_{k}\right)$ from every neighbouring particle of radius $r_{k}$ at a surface-to-surface distance $\delta_{k}$ from a central particle of radius $r_{\mathrm{c}}$ :

$$
V_{\text {cell }}=V_{\text {particle }}+\sum_{k=1}^{n} V^{*}\left(r_{\mathrm{c}}, r_{k}, \delta_{k}\right),
$$

where $V_{\text {particle }}=\frac{4}{3} \pi r_{\mathrm{c}}^{3}$ is the volume of the central particle.

Feature two will be satisfied by treating contacting neighbours differently from non-contacting. This will be achieved by assuming that all the contacts are at $\delta_{k}=0$ while all the noncontacting neighbours are at the same finite distance, $\delta_{k}=\delta$, which we take to be a fraction of the mean particle radius:

$$
\delta=\alpha\left(r_{\mathrm{c}}\right)\langle r\rangle .
$$

Here $\alpha\left(r_{\mathrm{c}}\right)$ is an additional adjustable parameter in our model. Its nature, however, is quite different from that of the available solid angle $\Omega_{\text {max }}\left(r_{\mathrm{c}}\right)$ or the contacting probability $p\left(r_{\mathrm{c}}\right)$, since $\alpha\left(r_{\mathrm{c}}\right)$ does not have a strong physical interpretation outside of this particular definition of the cell. In particular it is important to note that the parameter $\alpha\left(r_{\mathrm{c}}\right)$ should not be thought of as relating to a real physical distance between particles. It is rather a bookkeeping parameter with which to tune the definition of a local cell, in order to match model calculations with experimental measurements. In our previous work ${ }^{22}$ we fixed the value of the parameter $\alpha\left(r_{\mathrm{c}}\right)$ so that the mean local packing fraction obtained from the model matches the measured mean local packing fraction.

Let us introduce the volume $V^{*}$ added to the volume of a local cell due to the presence of a neighbour. We define this volume in the following way. Let $\mathscr{C}$ be the cone subtended on a central particle by a neighbouring particle in contact, and $\mathscr{S}$ be the 


$$
\begin{aligned}
V^{*}\left(r_{\mathrm{c}}, r, \delta\right)= & \frac{\pi(\delta+2 r) r_{\mathrm{c}}\left(2 r+r_{\mathrm{c}}\right)\left(\delta+2 r_{\mathrm{c}}\right)^{3}\left(-2 r_{\mathrm{c}}^{3}+2\left(\sqrt{r_{\mathrm{c}}\left(2 r+r_{\mathrm{c}}\right)}-r\right) r_{\mathrm{c}}^{2}+3 r^{2} r_{\mathrm{c}}+r^{2}\left(\delta+3 r-2 \sqrt{r_{\mathrm{c}}\left(2 r+r_{\mathrm{c}}\right)}\right)\right.}{24\left(\delta r_{\mathrm{c}}\left(2 r+r_{\mathrm{c}}\right)+\left(r+r_{\mathrm{c}}\right)\left(r_{\mathrm{c}}^{2}+2 r r_{\mathrm{c}}-\sqrt{r_{\mathrm{c}}\left(2 r+r_{\mathrm{c}}\right)} r_{\mathrm{c}}+r \sqrt{r_{\mathrm{c}}\left(2 r+r_{\mathrm{c}}\right)}\right)\right)^{2}} \\
& -\frac{2}{3} \pi\left(1-\frac{\sqrt{1+\frac{2 r}{r_{\mathrm{c}}}}}{1+\frac{r}{r_{\mathrm{c}}}}\right) r_{\mathrm{c}}^{3} .
\end{aligned}
$$

surface of the central particle. Let $\mathscr{H}^{\prime}$ be the surface of the hyperboloid defined by the navigation map of the central particle of radius $r_{\mathrm{c}}$ and a neighbour of radius $r$ with surfaces seperated by a distance $\delta$ (see Fig. 6b). The volume $V^{*}$ is then defined as the portion of the cone $\mathscr{C}$ between the central particles surface $\mathscr{S}$ and the hyperboloid $\mathscr{H}^{\prime}$. This volume is a function of $r_{\mathrm{c}}, r$ and $\delta$ given by:

Let us consider the generic situation of a given central particle of radius $r_{\mathrm{c}}$ surrounded by $n$ neighbours, among which $z$ are in contact. Let $\left\{r_{k}\right\}_{k=1 \ldots n}$ be the list of the neighbours' radii, arranged such that the $z$ first radii correspond to the contacts. The volume of the cell is given by:

$$
V_{\text {cell }}=\frac{4 \pi r_{\mathrm{c}}^{3}}{3}+\sum_{k=1}^{z} V^{*}\left(r_{\mathrm{c}}, r_{k}, 0\right)+\sum_{k=z+1}^{n} V^{*}\left(r_{\mathrm{c}}, r_{k}, \delta\right)
$$

\section{B. Local packing fraction distribution}

The approximation (31) of the volume of the cell permits us to estimate the local packing fraction. Using the notation described above the local packing fraction of a cell is simply defined as

$$
\phi_{1}=\frac{V_{\text {particle }}}{V_{\text {cell }}} .
$$

We can rewrite this relation in a way that makes the calculation of the distribution easier. For a given central particle of radius $r_{\mathrm{c}}$ surrounded by $n$ neighbours among which $z$ are contacts, let us introduce the random variable

$$
\psi_{1}=\frac{V_{\text {cell }}}{V_{\text {particle }}}-1 \geq 0
$$

Using eqn (6), this variable can be written as a sum,

$$
\psi_{1}=\sum_{k=1}^{z} \lambda\left(r_{\mathrm{c}}, r_{k}\right)+\sum_{k=1}^{n} \mu\left(r_{\mathrm{c}}, r_{k}\right),
$$

where,

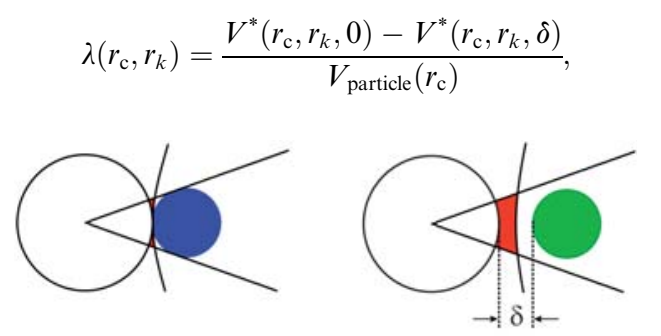

(a)

(b)

Fig. 6 Volume contributions $V^{*}$ (coloured in red) for contact (a) and neighbours at a surface-to-surface distance $\delta(b)$.

$$
\mu\left(r_{\mathrm{c}}, r_{k}\right)=\frac{V^{*}\left(r_{\mathrm{c}}, r_{k}, \delta\right)}{V_{\text {particle }}\left(r_{\mathrm{c}}\right)},
$$

and we used the notation $V_{\text {particle }}\left(r_{c}\right) \equiv V_{\text {particle }}=\frac{4}{3} \pi r_{c}^{3}$ to stress that this volume depends on $r_{\mathrm{c}}$. The variables $\lambda$ and $\mu$ are twopoint random variables whose distributions can be computed following the steps presented in Appendix A. The conditional probability density of $\psi_{1}$ for given $r_{\mathrm{c}}, z$ and $n$ can then be obtained following Appendix B. A simple change of variable,

$$
\phi_{1}=\frac{1}{\psi_{1}-1}
$$

allows then for the estimation of the local packing distribution for given $z, n$ and $r_{\mathrm{c}}, \Pi\left(\phi_{1} \mid z, n, r_{\mathrm{c}}\right)$. To obtain the distribution of the local packing fraction around a particle of radius $r_{\mathrm{c}}$, we need to average on $n$ and $z$, keeping in mind that these two variables are not independent. By construction, the probability density of the contact number $z$ for a given $n$ is a binomial distribution with success rate $p\left(r_{\mathrm{c}}\right), B\left(z ; n, p\left(r_{\mathrm{c}}\right)\right)$. We therefore have

$$
\begin{gathered}
\Pi\left(\phi_{1} \mid n, r_{\mathrm{c}}\right)=\sum_{z=0}^{n} B\left(z ; n, p\left(r_{\mathrm{c}}\right)\right) \Pi\left(\phi_{1} \mid z, n, r_{\mathrm{c}}\right), \\
\Pi\left(\phi_{1} \mid r_{\mathrm{c}}\right)=\sum_{n=0}^{\infty} \Pi\left(\phi_{1} \mid n, r_{\mathrm{c}}\right) P_{\mathrm{n}}\left(n \mid r_{\mathrm{c}}\right) .
\end{gathered}
$$

In the final expression, we use the neighbour number distribution for a given central particle radius, $P_{\mathrm{n}}\left(z \mid r_{\mathrm{c}}\right)$, obtained from eqn (20). For practical calculations, the summation in eqn (39) is truncated at a cut-off value for $n$ above which the distribution $P_{\mathrm{n}}\left(n \mid r_{\mathrm{c}}\right)$ is negligible. We can compute the probability map of the

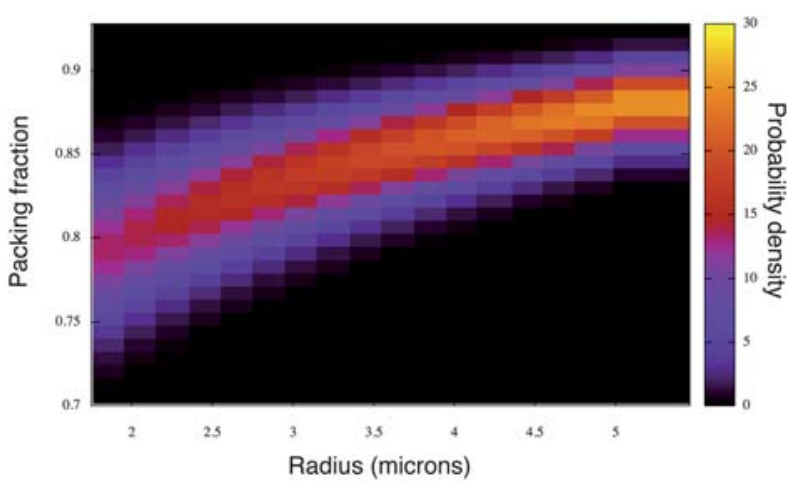

Fig. 7 Probability density map of the local packing fraction $\phi_{1}$, as a function of the central particle radius $r_{\mathrm{c}}$. Note that the packing is typically more efficient around large particles. 


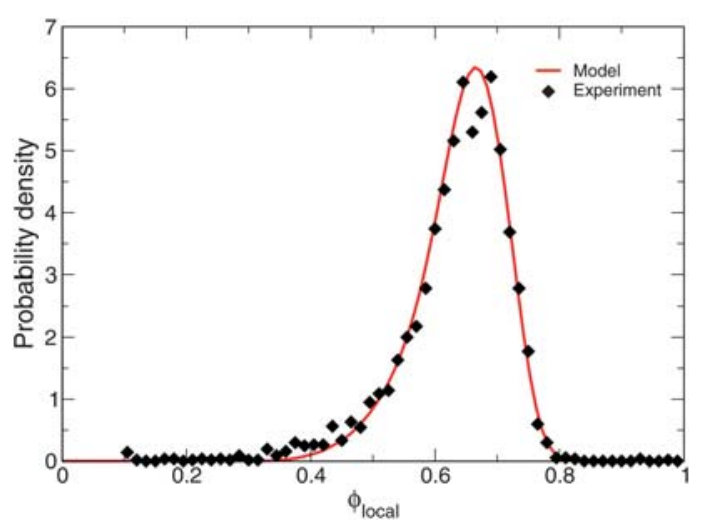

Fig. 8 Probability density of the local packing fraction.

local packing fraction, by a simple change of variables. This is presented in Fig. 7. To compare with experimental and numerical results, we can average over $r_{\mathrm{c}}$ to obtain the local packing fraction distribution in the sample.

The definition of the local packing fraction relies heavily on the definition of the local cell, and therefore on the parameter $\alpha$. In our work, this parameter is fixed so that the mean local packing fraction obtained by the model matches the mean local packing fraction measured in our numerical simulations. The result is presented in Fig. 8.

\section{Global packing fraction}

The global packing fraction is defined as

$$
\phi=\frac{V_{\text {total matter }}}{V_{\text {total }}}=\frac{\sum_{j=1}^{N} V_{\text {particle }}\left(r_{j}\right)}{\sum_{j=1}^{N} V_{\text {cell }}\left(r_{j}\right)},
$$

assuming that $V_{\text {total }}=\sum_{j=1}^{N} V_{\text {cell }}\left(r_{j}\right)$. In our model we can replace the spatial averages by ensemble averages, leading to

$$
\bar{\phi}=\frac{\left\langle V_{\text {particle }}\left(r_{\mathrm{c}}\right)\right\rangle}{\left\langle\left\langle V_{\text {cell }}\left(r_{\mathrm{c}}\right)\right\rangle\right\rangle} .
$$

The quantity in the denominator has to be averaged twice: first over the various configurations for a given $r_{\mathrm{c}}$ and then over $r_{\mathrm{c}}$ itself. Note that $\bar{\phi}$ is not the ensemble average of the local packing fractions:

$$
\bar{\phi} \neq\left\langle\phi\left(r_{\mathrm{c}}\right)\right\rangle P\left(r_{\mathrm{c}}\right)
$$

We can evaluate the global packing fraction $\bar{\phi}$ using Wald's identity ${ }^{45}$ to compute the mean cell volume for a given $r_{\mathrm{c}}$. Starting with the expression (31) of the cell volume written as

$$
\begin{aligned}
V_{\text {cell }}= & V_{\text {particle }}\left(r_{\mathrm{c}}\right)+\sum_{k=1}^{z}\left(V^{*}\left(r_{\mathrm{c}}, r_{k}, 0\right)-V^{*}\left(r_{\mathrm{c}}, r_{k}, \delta\right)\right) \\
& +\sum_{k=1}^{n} V^{*}\left(r_{\mathrm{c}}, r_{k}, \delta\right)
\end{aligned}
$$

we first average of the neighbours' radii leading to

$$
\begin{aligned}
\left\langle V_{\text {cell }} \mid r_{\mathrm{c}}\right\rangle & =V_{\text {particle }}\left(r_{\mathrm{c}}\right)+\left\langle n \mid r_{\mathrm{c}}\right\rangle\left(p\left(r_{\mathrm{c}}\right)\left\langle V^{*}\left(r_{\mathrm{c}}, r, 0\right) \mid r_{\mathrm{c}}\right\rangle\right. \\
& \left.+\left(1-p\left(r_{\mathrm{c}}\right)\right)\left\langle V^{*}\left(r_{\mathrm{c}}, r, \delta\right) \mid r_{\mathrm{c}}\right\rangle\right),
\end{aligned}
$$

where we used

$$
\left\langle z \mid r_{\mathrm{c}}\right\rangle=p\left(r_{\mathrm{c}}\right)\left\langle n \mid r_{\mathrm{c}}\right\rangle .
$$

The expression in eqn (44) can be averaged against $P\left(r_{\mathrm{c}}\right)$ to obtain the mean volume of a cell

$$
\left\langle\left\langle V_{\text {cell }}\left(r_{\mathrm{c}}\right)\right\rangle\right\rangle=\int \mathrm{d} r_{\mathrm{c}} P\left(r_{\mathrm{c}}\right)\left\langle V_{\text {cell }} \mid r_{\mathrm{c}}\right\rangle .
$$

The mean volume of a particle is simply given by

$$
\left\langle V_{\text {particle }}\right\rangle=\frac{4 \pi}{3} \int \mathrm{d} r_{\mathrm{c}} P\left(r_{\mathrm{c}}\right) r_{\mathrm{c}}^{3} .
$$

We finally obtain the global packing fraction as

$$
\bar{\phi}=\frac{4 \pi}{3} \frac{\int \mathrm{d} r_{\mathrm{c}} P\left(r_{\mathrm{c}}\right) r_{\mathrm{c}}^{3}}{\int \mathrm{d} r_{\mathrm{c}} P\left(r_{\mathrm{c}}\right)\left\langle V_{\text {cell }} \mid r_{\mathrm{c}}\right\rangle} .
$$

This expression allows us to compute the global packing fraction, starting from the distribution of radii only, once the model parameters $\Omega_{\max }\left(r_{\mathrm{c}}\right), p\left(r_{\mathrm{c}}\right)$ and $\alpha$ are self-consistently fixed.

\section{Limits of applicability of the model}

Our model works under the assumption that the packing is statistically homogeneous, such that the polydispersity around each particle in the packing can be described by the same radius distribution $P(r)$ as the overall packing. Using this assumption, the packing formation is described by two consecutive random processes. The first one corresponds to the formation of the set of neighbours around a given particle. The space around a particle of radius $r_{\mathrm{c}}$ is quantified by the available solid angle around it $\Omega_{\max }\left(r_{\mathrm{c}}\right)$. The formation of a set of nearest neighbours is modeled by the first passage of a one-dimensional random walk, where the steps are the solid angle subtended by the incoming particles on the central particle. We do not take into account the positions of the neighbours around the central particle, and we assume that each step in the random walk is independent and identically distributed. The number of neighbours $n$ is the number of steps in the walk before it reaches without crossing the maximum solid angle $\Omega_{\text {max }}$. A subset of particles in contact is then selected among the neighbours by a succession of Bernoulli trials with a probability of success $p\left(r_{\mathrm{c}}\right)$, assuming that there is no collective behaviour in this selection process. Although we neglect explicit correlation in the steps of the random walk and in the formation of contacts, it does not mean that the model neglects all correlations. Actually correlations are taken into account, at least partially, in the parameters of this effective model, namely the available solid angle $\Omega_{\max }\left(r_{\mathrm{c}}\right)$ and the contact probability $p\left(r_{\mathrm{c}}\right)$.

In order to describe a polydisperse packing we have introduced several fundamental assumptions:

A0 Long range disorder is dominated by local disorder due to the particle size distribution.

A1 The packing is homogeneous: the distribution of particle sizes around particles of size $r_{\mathrm{c}}$ is identical to the overall distribution of particle sizes.

A2 The neighbours are chosen independently in the random walk. 
A3 The contacts are chosen among the neighbours independently.

A4 A local cell about each particle can be defined as in section V.A.

Given these assumptions, our model allows for the calculation of the distributions of the coordination number, the number of neighbours and the local packing fraction.

In our previous study ${ }^{22}$ we found experimental evidence to justify two additional simplifications:

$\mathrm{S} 1$ The solid angle available to neighbours $\Omega_{\max }\left(r_{\mathrm{c}}\right)$ is independent of the central particle radius $r_{\mathrm{c}}$.

$\mathrm{S} 2$ The ratio of contacts to neighbours $p\left(r_{\mathrm{c}}\right)$ is independent of the central particle radius $r_{\mathrm{c}}$.

If any of the above assumptions A0-A4 become invalid then our model may not successfully describe the packing. However, if $\mathrm{S} 1$ and $\mathrm{S} 2$ no longer hold the model should still be successful but will require the dependence of model parameters on the radius $r_{\mathrm{c}}$. In the rest of this section we illustrate the limitations of this model with several examples.

\section{A. Monodisperse or slightly polydisperse packings}

If a packing is monodisperse then there is no local disorder due to the particle size distribution. Therefore assumption A0 is violated and our model breaks down. In such a case our model predicts $P\left(n \mid r_{c}\right)$ to be a Dirac distribution, while experimentally and numerically one finds a distribution with a finite width.

More generally, we expect our model to break down if the polydispersity is not strong enough to be the dominant source of disorder in the packing. To probe this limit we consider packings with log-normal size distributions with mean 1 and standard deviation $\sigma$. By reducing the value of $\sigma$ we can explore the crossover from polydisperse to monodisperse and observe how the models predictions gradually fail (Fig. 9). For this particular family of distributions the model becomes progressively worse for values of $\sigma$ below $\approx 0.09$. However, one should note that the

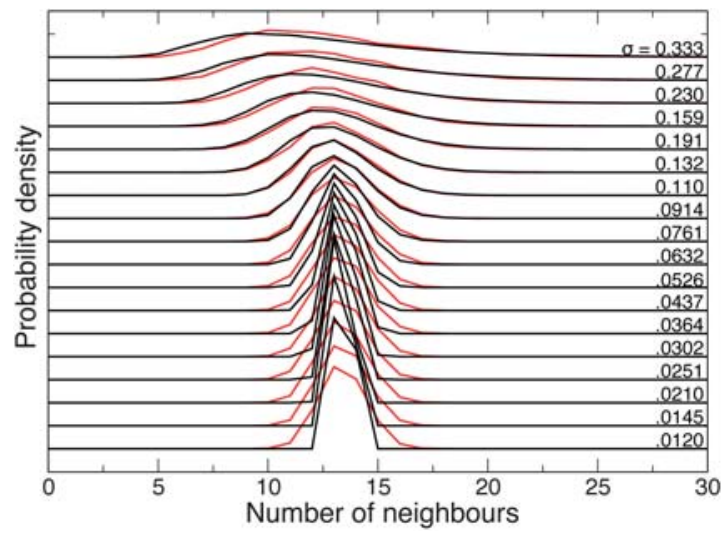

Fig. 9 Crossover from polydisperse distributions to monodisperse distributions probes the limits of applicability of the model. The probability distribution of number of neighbours is shown for packings with log-normal size distributions with mean $=1$ and standard deviation $\sigma$ as indicated. Curves shown in black are obtained from molecular dynamics simulations while those in red show model predictions. The agreement becomes progressively worse for values of $\sigma$ below $\approx 0.09$. crossover will depend on the details of the family of size distributions under consideration.

\section{B. Inhomogeneous packings}

If a packing is non-homogeneous then assumption A1 breaks down and the model no longer applies. In such a case each particle sees a local environment characterized by a size distribution $P(r, k)$ where $k$ is a variable describing the inhomogeneity. In such a case the $k$ dependence would have to be incorporated into a new model to describe the packing. This may not always be possible to do.

One common form of inhomogeneity is size segregation in packings. Such segregation often arises from buoyancy effects in emulsion and colloidal systems or "Brazil nut"-like effects in granular packings. In a layered size-segregated packing, even though the system taken as a whole may be strongly polydisperse each layer is only weakly polydisperse, with a radius depending on the layer position. In this case the $k$ parameter will be the layer position $h$ such that the polydispersity is described by $P(r, h)$. If this $h$ dependence is propagated through the model it should be able to describe the packing in each layer as $P(n \mid h), P(z \mid h), P(\phi \mid h)$.

Another, less tractable, form of size segregation arises in bidisperse packings with large size ratios in which the majority of the volume is filled by large particles. Such a packing will consist of a network of large particles decorated by small particles in the voids. Most small particles will be in a local environment dominated by small particles. Therefore the particle size distribution about a given particle will depend on that particle's size and the overall geometry of packing. The propagation of this form of size segregation through the model would require an a priori knowledge of the packing structure, rendering the model superfluous.

\section{Bidisperse packings}

It is easy to imagine packings for which the simplifications $\mathrm{S} 1$ and $\mathrm{S} 2$ will not hold. Simplification S1 fails in the regime of bidisperse packings where the volume fraction of small particles is less than that of large particles. ${ }^{46}$ In this regime the small particle effectively decouple from the packing with a majority becoming "floaters" or "rattlers". Therefore the ratio of contacts to neighbours $p(r)$ about a small particle must necessarily be smaller around a small particle than around a big particle. Since a floater has on average a lower packing fraction than a particle with many contacts the effect will be that our model overestimates the packing fraction in this regime, leading to a packing fraction larger than the infinite size ratio upper limit. ${ }^{47}$ However, by abandoning the simplification that $p(r)$ is a constant independent of $r$ it should be possible to accurately describe the packing and predict the value of packing fraction in this regime.

\section{Conclusion}

In this article we have presented a model with which to describe random polydisperse packings of frictionless spheres by characterizing the distributions of nearest neighbours, contacts, and local packing fraction. In our model, a stable random packing is created in two stages at the single particle level. First, the available space around each particle is filled by a set of nearest neighbours. Second, mechanical equilibrium is achieved by choosing some of these 
neighbours to be in contact with the central particle. As in any effective model there are necessarily limitations to applicability. The fundamental condition for this model to function is that the polydispersity is the dominant source of disorder in the packing. Therefore, this model can not be applied to packings which are highly monodisperse. As presently constructed, this model can not describe inhomogeneous packings. Further, some of the simplifications must be abandoned to accurately characterize bidisperse packings in the "floater" regime. The applicability of this model, however, can be extended at the cost of adding additional parameters. For example, this model can be extended to non-homogeneous packings by first identifying the variable upon which the non-homogeneity depends (e.g. height for size segregated samples) and then running all model calculations predicated on that variable.

Turning the granocentric model into a complete theory would require a computation of the parameters in the model from first principles given only a radius distribution as the starting point. Such a program is far beyond the scope of the present paper but might be an interesting path to follow to gain a better understanding of the random packing of spheres.

As constructed, this model is a purely statistical description of packings. However, it is strongly suggestive of a dynamical process for the formation of packings. This provides an impetus to pursue an extension of this model to describe this dynamical formation.

\section{Appendix A: 2-Point random variable}

The "granocentric" point of view used to construct the model allows for all quantities to be dependent on the radius of the central particle. As a result, most of the physical quantities involved in the model are two-point quantities, depending both on the radius of the central particle and on the radius of a neighbour. In particular, this is the case for the solid angle subtended on the central particle by a neighbour, defined by eqn (8). Let us consider the generic case of a physical quantity $\phi\left(r, r_{\mathrm{c}}\right)$. The randomness coming from the radius distribution $P(r)$ propagates to $\phi$ both through $r$ and $r_{\mathrm{c}}$. The description of the resulting randomness is achieved using a conditional probability distribution.

Consider a given central particle, of radius $r_{\mathrm{c}}$. The physical quantity $\phi\left(r, r_{\mathrm{c}}\right)$ is then a function of one variable $(r)$. If this reduced function defines a change of variable then the conditional distribution of $\phi$ for a given $r_{\mathrm{c}}, \Pi\left(\phi \mid r_{\mathrm{c}}\right)$, is obtained from $P(r)$ by

$$
\Pi\left(\phi \mid r_{\mathrm{c}}\right)=\frac{1}{d \phi / d r} P\left(r\left(\phi, r_{\mathrm{c}}\right)\right) .
$$

This expression is used, for instance, to compute the solid angle distribution around a given central particle $f_{\mathrm{c}}(\omega)$, which in turn is used to compute the contact and neighbour distributions around a particle of radius $r_{\mathrm{c}}$. From this conditional distribution, the distribution of $\phi$ can be obtained by

$$
\Pi(\phi)=\int \mathrm{d} r_{\mathrm{c}} \Pi\left(\phi \mid r_{\mathrm{c}}\right) P\left(r_{\mathrm{c}}\right) .
$$

\section{Appendix B: Sum of random variables}

In the model, some physical quantities appear as sums of other random variables, as is the case for the total solid angle occupied by neighbours around a central particle, or the local volume of a cell defined in eqn (31). We assume that the summed variables are independent, in order to use the following property. If $x$ and $y$ are two independent random variables distributed according to $P_{x}$ and $P_{y}$ respectively, then their sum $x+y$ is a random variable, distributed according to $P_{x+y}=P_{x} \star P_{y}$, where $\star$ is the convolution product. In practice, the calculation of $P_{x+y}$ is done by taking the Laplace transform of the previous relation, turning the convolution product into a simple product:

$$
\mathscr{L}\left[P_{x+y}\right]=\mathscr{L}\left[P_{x}\right] \times \mathscr{L}\left[P_{y}\right] .
$$

As a simple extension, let us consider the particular case of $y=\sum_{k=1}^{n} x_{k}+\sum_{j=1}^{z} w_{j}$, where $x_{k}$ and $w_{k}$ are independent, and all the $x_{k}$ are distributed according to $P_{x}$ and the $w_{k}$ according to $P_{w}$. Then the Laplace transform of $\Pi_{n, z}(y)$, the distribution of $y$ for a given $n$ and a given $z$ is obtained from:

$$
\mathscr{L}\left[\Pi_{n, z}\right]=\mathscr{L}\left[P_{x}\right]^{n} \times \mathscr{L}\left[P_{w}\right]^{z} .
$$

Such an extension is useful to compute the local packing fraction distribution, for a central particle with $n$ neighbours among which $z$ are in contact, as defined in eqn (31).

\section{Acknowledgements}

The authors would like to thank Gérard Ben Arous, Ivan Z. Corwin and Jean-Baptiste Gouéré for useful discussions. This work was partially supported by NYU MRSEC Award DMR:0820341. J. B. Holds a Career Award at the Scientific Interface from the Burroughs Wellcome Fund.

\section{References}

1 S. Hales. Vegetable staticks, W. and J. Innys, London, 1727.

2 A. O. N. Siemens and M. van Hecke, Jamming: A simple introduction, Physica A: Statistical Mechanics and its Applications, 2010, DOI: 10.1016/j.physa.2010.02.027.

3 T. S. Majumdar, M. Sperl, S. Luding and R. P. Behringer, The jamming scenario an introduction and outlook, Phys. Rev. Lett., 2007, 98, 058001 .

4 M. E. Cates, J. P. Wittmer, J.-P. Bouchaud and P. Claudin, Jamming, force chains, and fragile matter, Phys. Rev. Lett., 1998, 81(9), 18411844.

5 A. J. Liu and S. R. Nagel, Nonlinear dynamics: Jamming is not just cool anymore, Nature, 1998, 396, 21.

6 D. Howell, R. P. Behringer and C. Veje, Stress fluctuations in a 2d granular Couette experiment: a continuous transition, Phys. Rev. Lett., 1999, 82(26), 5241-5244.

7 V. Trappe, V. Prasad, L. Cipelletti, P. N. Segre and D. A. Weitz, Jamming phase diagram for attractive particles, Nature, 2001, 411(6839), 772-775.

8 E. I. Corwin, H. M. Jaeger and S. R. Nagel, Structural signature of jamming in granular media, Nature, 2005, 435, 1075.

9 L. E. Silbert, D. Ertaş, G. S. Grest, T. C. Halsey and D. Levine, Analogies between granular jamming and the liquid-glass transition, Phys. Rev. E: Stat., Nonlinear, Soft Matter Phys., 2002, 65(5), 51307.

10 N. Lacevic and S. C. Glotzer, Dynamical Heterogeneity and Jamming in Glass-Forming Liquids, J. Phys. Chem. B, 2004, 108(51), 1962319633.

11 A. J. Liu and S. R. Nagel, editors. Jamming and rheology: constrained dynamics on microscopic and macroscopic scales. Taylor \& Francis, 2001.

12 A. Coniglio, A. Fierro, H. J. Herrmann, and M. Nicodemi, editors. Unifying concepts in granular media and glasses. Elsevier Science, 2004. 
13 G. Parisi and F. Zamponi, Mean-field theory of hard sphere glasses and jamming, Rev. Mod. Phys., 2010, 82(1), 789-845.

14 S. F. Edwards and R. B. S. Oakeshott, Theory of powders, Phys. A, 1989, 157(3), 1080.

15 F. Lechenault, F. da Cruz, O. Dauchot and E. Bertin, Free volume distributions and compactivity measurement in bidimensional granular packing, J. Stat. Mech.: Theory Exp., 2006, 2006, P07009.

16 T. Aste, Volume fluctuations and geometrical constraints in granular packs, Phys. Rev. Lett., 2006, 96, 018002.

17 C. Song, P. Wang and H. A. Makse, A phase diagram for jammed matter, Nature, 2008, 453, 629 .

18 E. Santiso and E. A. Muller, Dense packing of binary and polydisperse hard spheres, Mol. Phys., 2002, 100(15), 2461-2469.

19 L. Pournin, M. Ramaioli, P. Folly and T. M. Liebling, About the influence of friction and polydispersity on the jamming behavior of bead assemblies, Eur. Phys. J. E, 2007, 23(2), 229-235.

20 M. van Hecke, Jamming of Soft Particles: Geometry, Mechanics, Scaling and Isostaticity, J. Phys.: Condens. Matter, 2010, 22 033101.

21 A. Donev, I. Cisse, D. Sachs, E. A. Variano, F. H. Stillinger, R. Connelly, S. Torquato and P. M. Chaikin, Improving the density of jammed disordered packing using ellipsoids, Science, 2004, 303, 990.

22 M. Clusel, E. I. Corwin, A. O. N. Siemens and J. Brujić, A "granocentric" model for random packing of jammed emulsions, Nature, 2009, 460(7255), 611-615.

23 M. Hermes and M. Dijkstra, Jamming of polydisperse hard spheres, Europhys. Lett., 2010, 89, 38005.

$24 \mathrm{H}$. J. H. Brouwers, Particle-size distribution and packing fraction of geometric random packings, Phys. Rev. E: Stat., Nonlinear, Soft Matter Phys., 2006, 74(3), 31309.

25 N. Ouchiyama and T. Tanaka, Porosity estimation for random packings of spherical particles, Ind. Eng. Chem. Fundam., 1984, 23(4), 490-493.

$26 \mathrm{~N}$. Ouchiyama and T. Tanaka, Porosity estimations of mixed assemblages of solid particles with different packing characteristics, J. Chem. Eng. Jpn., 1988, 21(2), 157-163.

27 T. Stovall and D. E. Larrad, Linear packing density model of grain mixtures, Powder Technol., 1986, 48(1), 1.

28 A. B. Yu and N. Standish, Porosity calculations of multi-component mixtures of spherical particles, Powder Technol., 1987, 52(3), 233-241.

29 J. A. Dodds, Simplest statistical geometric model of simplest version of multicomponent random packing problem, Nature, 1975, 256(5514), 187-189.
30 J. A. Dodds and H. Kuno, Computer-simulation and statistical geometric model for contacts in a binary random 2-dimensional disk packings, Nature, 1977, 266(5603), 614-615.

$31 \mathrm{~J}$. A. Dodds, The porosity and contact points in multicomponent random sphere packings calculated by a simple statistical geometric model, J. Colloid Interface Sci., 1980, 77(2), 317-327.

32 B. Bideau and J. A. Dodds, editors. Physics of granular media, Les Houches series, Nova Science Publishers, 1991.

33 S. Alexander, Amorphous solids: their structure, lattice dynamics and elasticity, Phys. Rep., 1998, 296, 65.

34 C. F. Moukarzel, Isostaticity in granular matter, Granular Matter, 2001, 3(1-2), 41-52.

35 E. N. Gilbert, Random subdivisions of space into crystals, Ann. Math. Stat., 1962, 33(3), 958-972.

36 N. N. Medvedev, Application of the Voronoi-Delone method to description of structure of intersphere space in polydisperse systems, Dokl. Phys. Chem., 1994, 337(1-6), 157-161.

37 P. Richard, L. Oger and J.-P. Troadec, A model of binary assemblies of spheres, Eur. Phys. J. E, 2001, 6(4), 295.

38 G. Voronoi, Nouvelles applications des paramètres continus à la théorie des formes quadratiques, J. Reine Angew. Math., 1907, 133, $97-178$.

39 J. Brujić, C. Song, P. Wang, C. Briscoe, G. Marty and H. A. Makse, Measuring the coordination number and entropy of a $3 \mathrm{~d}$ jammed emulsion packing by confocal microscopy, Phys. Rev. Lett., 2007, 98(24), 248001.

40 S. Plimpton, Fast parallel algorithms for short-range molecular dynamics, J. Comput. Phys., 1995, 117(1), 1-19.

41 C. S. O'Hern, L. E. Silbert, A. J. Liu and S. R. Nagel, Jamming at zero temperature and zero applied stress: The epitome of disorder, Phys. Rev. E: Stat., Nonlinear, Soft Matter Phys., 2003, 68(1), 011306.

42 A. Mallet. Numerical Inversion of Laplace Transform, 2000, Mathmatica package, http://library.wolfram.com/infocenter/ Mathsource/2691/.

43 T. Aste, M. Saadaftar and T. J. Senden, Local and global relations between the number of contacts and density in monodisperse sphere packs, J. Stat. Mech.: Theory Exp., 2006, 2006, P07010.

44 T. Aste and T. Di Matteo, Emergence of Gamma distributions in granular materials and packing models, Phys. Rev. E: Stat., Nonlinear, Soft Matter Phys., 2008, 77, 021309.

45 R. Durrett. Probability: theory and examples. Duxbury Press, 1995.

46 R. S. Farr and R. D. Groot, Close packing density of polydisperse hard spheres, J. Chem. Phys., 2009, 131, 244104.

47 A. V. Kyrylyuk1 and A. P. Philipse, Private communication, 2009. 